\title{
Thermo-mechanical analysis of a toughened thermosetting system
}

Mauro Zarrelli ${ }^{1}$, A.A. Skordos $^{2}$ and Ivana K Partridge ${ }^{2}$

${ }^{1}$ Institute of Composite and Biomedical Materials (IMCB) - Research National Council (CNR), P.le E. Fermi, Portici 8055 (Naples) Italy

${ }^{2}$ Advanced Materials Dept, Cranfield University, Cranfield, Bedford, MK43 0AL, UK

Corresponding author: $\quad$ Dr Mauro Zarrelli

IMCB - Institute of Composite and Biomedical Materials

CNR - Research National Council

Piazzale e. Fermi, Portici (Naples), Italy

E- Mail: mauro.zarrelli@imcb.cnr.it 


\section{ABSTRACT}

This paper reports experimental results of viscoelastic mechanical tests, at five different levels of conversion, for a thermoset composite matrix system toughened with an appropriate percentage of thermoplastic polymer. Results from static tests are used to build master curves at specific degree of cure while shift factors are compared with corresponded values from dynamic experiments in order to assess the validity of time temperature superposition for each conversion. Neat resin plates have been accurately cured, according to the full kinetics model for dynamic and isothermal temperature regime; conversion gradient within the plane and through the thickness was assessed by thermal analysis of samples taken from different locations before extracting the samples from the plates.

Viscoelastic behaviour of the resin matrix shows sensible difference in relaxation time spectrum with the conversion according to the provisional trend of mobility theory; higher conversion induces horizontal shifting of the principal relaxation time for each level of conversion which can be very well related with glass transition at same conversion. Good results have been also obtained for the ultimate modulus of the resin at temperature just before the starting of the co-curing phase for the partially cured samples.

KEYWORDS: mechanical property, viscoelasticity, time-temperature superposition, conversion, thermoset 


\section{INTRODUCTION}

There has been limited work reported in the literature on the development of viscoelastic mechanical properties in curing thermosets. Evolution of the mechanical properties with the increasing degree of cure is a very important effect to monitor in order to provide adequate mathematical model for process simulation software.

As the thermosetting resin cures, its material characteristics change from the behaviour of a viscous liquid (low stiffness) material, in its uncured state, to an elastic rigid solid (high stiffness), in its fully cured state. The mechanical properties of this resin phase are governed by competing mechanisms of chemical kinetics hardening, due to the polymerization, and viscoelastic relaxation phenomena, associated with the high processing temperatures. At elevated temperature and near the glass transition temperature, the system is characterised by a strongly viscoelastic behaviour with cure dependent relaxation times as the cure progresses; when the temperature is lowered, the elastic behaviour is dominant, with no sensible relaxation effect.

Considering that temperature gradient inside the part and an associated degree of conversion gradient lead to a sort of complex "mapping" of the mechanical properties, suitable predictive models are necessarily required to achieve accurate residual stress analysis. Many efforts have been focused on the development of relationships between viscoelastic properties and specific polymer characteristics, such as molecular weight, molecular weight distribution, and degree of branching [1]. White and Mather [2] investigated the effect of cure on viscoelastic properties using ultrasonic techniques, while dielectric techniques have been used to characterize curing polymers [3] by relating ionic mobility to dynamic viscosity. Suzuki et al. [4] presented relaxation data for epoxies cured according to various cure cycles. However, in this analysis, the cure states of these samples were not determined. Therefore, relationship between mechanical properties and cure evolution could not be properly explored. In work by Kim and Hahn [5], the progression of the elastic modulus of thermoset resins has been reported to have a linear relationship with the degree of cure in the region of liquid-solid transformation. Outside this region two fixed values were assumed, respectively for the elastic modulus of uncured and fully cured system. Kim and White reported a systematic analysis of the effect of cure state on the stress relaxation modulus, the relaxation spectrum and the glass transition temperature [6,7]. Recently, Simon et al. [8] have presented an interesting research work on cure-dependent storage modulus for a commercial toughened epoxy resin. Giving a general methodology to model the time-temperature-conversion effects of viscoelastic 
response of thermosets, the predictions of the shear modulus for a general temperature history profile are presented. Based on the same time-cure superposition and extended to a more general concept of time-cure-temperature superposition principles is the methodology presented by Manson et al. [9] used to predict the viscoelastic properties of an high ultimate glass transition temperature epoxy resin at any stage during complex cure cycles. While experimental data in all the above cited works are limited to the post-gelation region, in O'Brien et al. [10] a complete understanding of the mechanism that drive the development of material properties during the cure is presented by a series of experiments on the unreacted or partially reacted liquid resin as well as on the post-gelled resin. The authors have used interrelationships between viscoelastic properties obtained by both dynamic and static experiments, depending on the handling state of the resin system, to evaluate one mechanical property from another one. Master curves for creep compliance and for storage and loss modulus were constructed and their shift factors analysed.

In this work, neat resin specimens were manufactured at various post-gelation degrees of conversion and tested under static stress relaxation and dynamic mode at different temperatures. Using raw data from static tests, master curves and shift factors were derived applying the time-temperature superposition principle. Obtained shift factors are then backapplied to dynamic raw data from dynamo-mechanical tests performed on the same samples to proof validity of the master curve procedure.

\section{MATERIAL AND EXPERIMENTS}

\subsection{Materials and sample preparation}

The main system considered in the present work is a TGDDM based resin, with catalyzed DDS cure and containing about $25 \%$ by weight of thermoplastic. The resin appears as translucent paste at room temperature with quite high viscosity. According to the manufacturer, the shelf life at $-18^{\circ} \mathrm{C}$ is 10 months and the glass transition temperature for the fully cured system is about $200^{\circ} \mathrm{C}$. No more details were given for this system, since it is not yet available on the market.

Resin plates were cast using the mould shown schematically in Fig. 1. Handling problems related with resin flow as well as the generation of excessive degree of cure gradients within the sample are the major considerations in designing the moulding procedure. A thin layer of 
PTFE/glass release film was stuck to each of the two glass plates, using PTFE tape at each end. The use of PTFE enabled easier release of the samples from the mould. In some cases, also liquid release agent (FREEKOTE 700) was used. The two metal bars were separated by a spacer of either rubber or aluminium, in which a reservoir was cut off. The spacer was held in place using double side PTFE tape on one mould and then the two moulds were held together using metallic jig clamps.

Five different plates of partially cured resin (dimension $200 \times 400 \times 2.5 \mathrm{~mm}$ ) were prepared under isothermal conditions $\left(180^{\circ} \mathrm{C}-160^{\circ} \mathrm{C}\right)$ for different periods, in order to achieve partially cure material at various extents. They were classified by labels A, B, C, D, E, with degree of conversion increasing from A to E. For sample labelled as E, full conversion was expected, based on the results of cure kinetics modelling.

\subsection{Sample conversion assessment by Differential Scanning Calorimetry}

Since the aim of the mechanical characterization was to acquire the necessary raw data to build the corresponding "master curves" at a particular degree of conversion, uniformly cured samples were required. According to results of the cure kinetics model ${ }^{11}$ different samples were prepared with accurate temperature profile and off-line measurements were also performed on each sample to verify the uniformity of conversion for each specimen both in plane and through thickness.

Figure 2 reports a schematic representation of the specimens' location on one side. All samples, of about 3-4 mg, were scanned by DSC from room temperature to $340^{\circ} \mathrm{C}$ at a heating rate of $10^{\circ} \mathrm{C} / \mathrm{min}$ to evaluate both the residual heat of reaction and the glass transition temperature. These two values provide useful information for evaluating the degree of conversion for each specimen and therefore they can be used to quantify the level of uniformity of conversion reached by the whole plates. The glass transition temeprature was determined as the inflection point of the heat flow step in the DSC signal in a thermal scan at $10 \mathrm{~K} \mathrm{~min}^{-1}$ for the isothermally cured samples; while for the dynamically cured specimens the same heating rate was used in order to avoid discrepancies due to rate dependence of glass transition. DSC tests were performed on 10 different samples taken from the same rectangular plate, respectively from each of the two sides to evaluate through-the-thickness and on each side, from the four corners and from the middle position, to evaluate in-plane degree of conversion gradient. 


\subsection{Dynamic Torsional Rheometric Tests}

Rheometric test using a torsional clamp system for solid samples were performed on partially cured materials. Specimens were cut from the plates after the degrees of conversion were assessed, using a low speed saw wafer machine. The cell mounted on the AR2000 rheometric equipment from TA Instruments is an Environmental Test Chamber (ETC) with thermal controlling system in the range $-150^{\circ} \mathrm{C}$ to $600^{\circ} \mathrm{C}$ with maximum heating rate of $15^{\circ} \mathrm{C} / \mathrm{min}$. All solid torsional tests were performed according to the specifications of ASTM D4065.

\subsection{Static and dynamic 3 point bending tests}

Stress relaxation and dynamical mechanical tests were performed by a TA-2940 DMA machine on rectangular strips taken from each of the partially cured plate. For the dynamic tests, specimens with dimensions of $50 \times 12 \times 3 \mathrm{~mm}$ were cut from each plate and subjected to frequency sweep tests between $0.01 \mathrm{~Hz}$ and $100 \mathrm{~Hz}$ at different isothermal temperatures under three-point bending test configuration. Each sample was equilibrated for about 5 minutes before test segment starting at each temperature, to provide a uniform temperature distribution inside the material during the frequency sweep. A total of 22 step-and-hold segments (between 25 and $320^{\circ} \mathrm{C}$ ) were considered in the machine program for all tests. However, since the partially cured resin had a glass transition temperature of $104^{\circ} \mathrm{C}$ for the least cured plate (A) and $181^{\circ} \mathrm{C}$ for the almost totally cured plate (E), only data in the region $25^{\circ} \mathrm{C}$ to $220^{\circ} \mathrm{C}$ have been used to build the corresponding dynamic master curves. When the material starts to react further, the test results are meaningless because of the changing degree of conversion. The same instrument was used to analyse the viscoelastic behaviour in the post-gelation region by 3 points bending under stress relaxation mode. In a simple stress relaxation experiment, the material specimen is subjected initially to a constant strain and the timedependent stress response is observed. In order to set the experiment on stress level within the range of linear viscoelasticity, some preliminary experiments have been performed with a fixed load rate. Final static tests, step-and-hold segments were performed at different temperatures up to the correspondent glass transition temperature. Three specimens were tested for each degree of conversion. After clamping, the specimens were equilibrated for 30 $\min$ at $30^{\circ} \mathrm{C}$ and then deformed for $40 \mathrm{~min}$. Afterwards, the temperature was increased 
$5-15^{\circ} \mathrm{C}$ and data acquisition was triggered. Above the glass transition temperature the test was stopped because of the onset of the post cure reaction.

\section{EXPERIMENTAL RESULTS}

\subsection{Thermal analysis results}

Calorimetric analysis of preliminary cast plates has revealed a high variability of degree of cure within the sample to invalidate further investigation, as mechanical testing or thermomechanical analysis. For these reasons, the determination and the minimization of the spread in the degree of conversion for each sample used for the dynamic and static mechanical tests has been an important issue to deal with during the preliminary stage of sample preparation. Since the aim of the mechanical characterization was to acquire the necessary raw data to construct the corresponding "master curves" at a particular degree of conversion, uniformly cured samples were required.

Figure 3 reports DSC scans for material taken from the least cured plate studied (labelled A); samples were extracted from the four corners and from the centre of this plate in order to study the in-plane conversion gradient. A value of $104^{\circ} \mathrm{C}$ for the glass transition temperature was found as the average value of all five measurements. Using the DiBenedetto glass transition model developed for the resin system of this study by Zarrelli et al. [11] the value of degree of cure corresponding to $T_{g}=104^{\circ} \mathrm{C}$ was found to be 0.68 . The same type of analysis was performed for the plate made of resin, type B, C, D, and E [12]. The degree of cure were also checked using the residual heat generation values and compared with resin kinetic model ${ }^{12}$, in order to determine the actual degree of conversion at the location from which the specimen was taken. Average values for the glass transition temperature, for residual heat of reaction and for the corresponding degree of conversion for all five partially cured plates (A-B-C-D-E) are reported in table 1. For plate, E the analysis of the heat flow profile has shown more uniform results with a very low value of variance for the residual heat of reaction. Figure 4 reports the non-reversible and reversible signal obtained by testing sample from plate labelled E (fully cured) by means of modulated dynamic scanning calorimetry (MDSC). 


\subsection{Solid rheometry results}

From each plate, five sets of three samples each were obtained by cutting and polishing. One of the sets was used to perform solid rheometric tests in order to verify the glass transition temperature and hence to assess the degree of conversion reached by the resin plates. The values obtained for the glass transition temperatures are higher than the corresponding values calculated using DSC technique. The difference can be attributed to the finite dimensions of the specimens. Negligible variation in degree of conversion has been found using the mechanically evaluated values of $T_{g}$. Figure 5 reports the results of dynamic rheometric scans on all five specimens. Here, the $T_{g}$ is defined as the temperature corresponding to the maximum value of $\tan \delta$, no experimental data could be obtained at degrees of cure lower than the level reached for plate A, due to the inherent fragility of the sample associated with the near gelation conversion, during the clamping or initial deformation of the sample.

\subsection{Static and Dynamic results on solid samples}

All the experimental data presented in this section are averaged over three different experiments. In the case of static experiments, depending upon the temperature range (from room temperature to the sample's glass transition temperature) and step increment, the time required to complete each test was between $300 \mathrm{~min}$ and $800 \mathrm{~min}$. Raw data for the case of $\alpha=0.90$, are reported in Fig. 6. The relaxation moduli for the entire partially cured sample are reported in Fig.6 and Fig. 7 as raw data. The temperature ranges from $25^{\circ} \mathrm{C}$ to the $T_{g}$ of the corresponding sample as measured by the torsional rheometer tests. For all the partially cured samples, the approach of the glass transition is clearly reflected by a sharp drop in the modulus. At the lowest temperatures, all the samples show glassy behaviour with an average modulus of about 3.4GPa; this value was treated as constant during the subsequent fitting procedure. Near the $T_{g}$, the rubbery modulus reached a plateau value ranging from $1.38 \mathrm{MPa}$ for the least cured sample to $60.9 \mathrm{MPa}$ for the fully cured resin samples.

It is important to emphasise that as the testing temperature is raised through the glass transition temperature, the raw data acquired become meaningless because of the onset of the post cure reaction. The raw data obtained were used to construct the relaxation modulus master curves up to the corresponding sample $T_{g}$ according to the time-temperature 
superposition principle with temperature of $T_{\text {ref }}=32^{\circ} \mathrm{C}$ was used as the reference temperature with no vertical shifts. Figure 9 reports all smoothed experimental master curves as used for further analysis.

\subsection{Master curves and shift factors: results}

For viscoelastic materials, the mechanical response is history-dependent and involves the use of a reduced time. Reduced time can be written using the temperature-cure dependent shift factor, $a_{T}(\alpha, T)$ from the integral in the following form:

$$
\xi=\frac{t}{a_{T}(\alpha, T)}
$$

where $\xi(t, \alpha, T)$ is the reduced time, $t$ is the actual experimental time and $a_{T}(\alpha, T)$ is the temperature-conversion shift factor value. For polymeric materials, shift factors are generally functions of temperature; in the case of a reactive system the dependence on the conversion needs to be taken into account in implementing the model. Fig. 10 shows the horizontal shift factors used to generate the master curves of stress relaxation modulus.

The application of the reduced variable method needs to be verified for the analogous data obtained under dynamic conditions. Using the same values of shift factor, master curves of dynamic storage and loss modulus were generated from raw data obtained from dynamic tests based on frequency sweeps. All these curves for the almost fully cured samples (label E) are shown in figs. 11 and 12.

The curves were built using $T=30^{\circ} \mathrm{C}$ as the reference temperature and no vertical shift was involved. At the lowest frequencies, storage modulus shows glassy behaviour with a linear variation of the modulus. O'Brien ${ }^{10}$ obtained the same behaviour for a different epoxy resin. At very low frequencies, corresponding to high temperatures of the raw testing data, values of the moduli cannot be acquired because of the inherent onset of the post cure reaction. Similar proof tests for the shift factor values have been performed for all five levels of conversion, and give satisfactory results in terms of dynamic master curves (see figure 13). In order to predict the evolution of the viscoelastic mechanical properties of the neat resin during cure, the shift factor curves need to be modelled with respect to both temperature and the degree of cure. 


\section{RESULTS AND DISCUSSION}

The viscoelastic mechanical properties of the neat resin system were investigated at different degrees of conversion. Five different levels of conversion have been considered, the resin plates being cured under appropriate conditions, as defined by the kinetic data presented previously $^{13}$.

Using differential mechanical analysis, stress relaxation measurements based on step loading and holding tests have been made on samples cut from partially cured resin plates. Master curves have been drawn from the raw data and the shift factors obtained have been verified for the dynamic data. Results show that appropriate master curves can be drawn for each degree of conversion and typical relaxation time can be evaluated. As expected, principal relaxation time are right shifted along the time axis as the cure advances. This is a typical behaviour as the more complex structure necessary induced longer time for the viscoleastic relaxation of the modulus.

\section{ACKNOWLEDGE}

This work was funded by the EPSRC (GR/M 89454). 


\section{FIGURES}

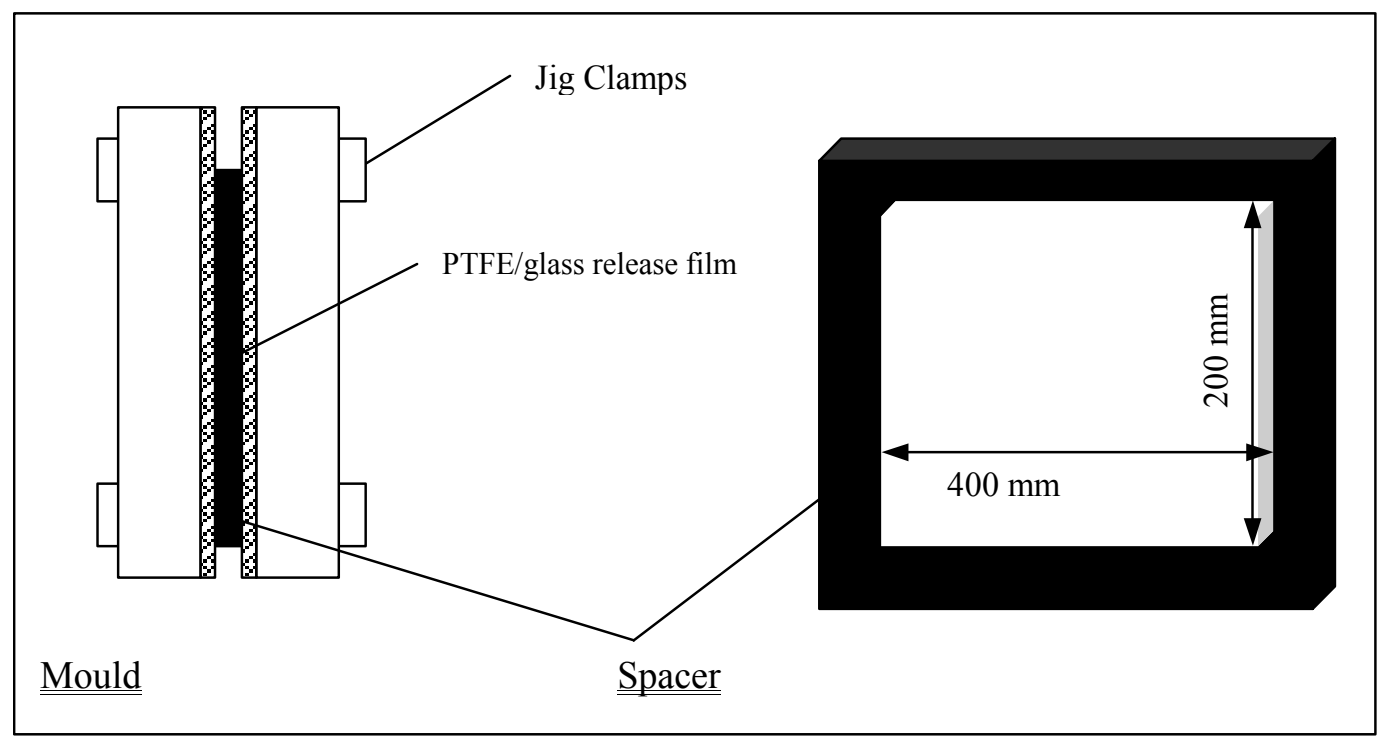

Fig. 1 Mould assembly for mechanical test samples (dimensions given for single resin plate)

Side 1 corner 1

Side 1 , centre location

Side 1 corner 3

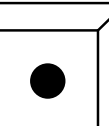

Side 1 corner 1

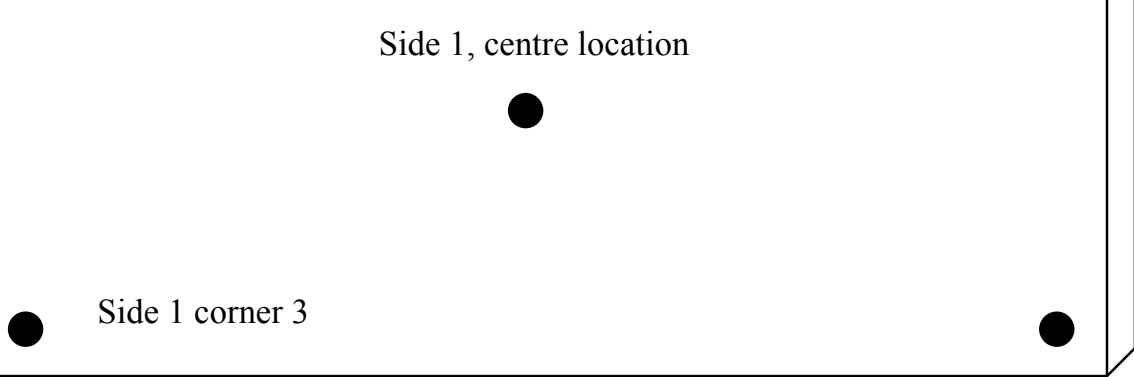

Fig. 2 Schematic neat resin plate with location of DSC sample used for quality inspection 


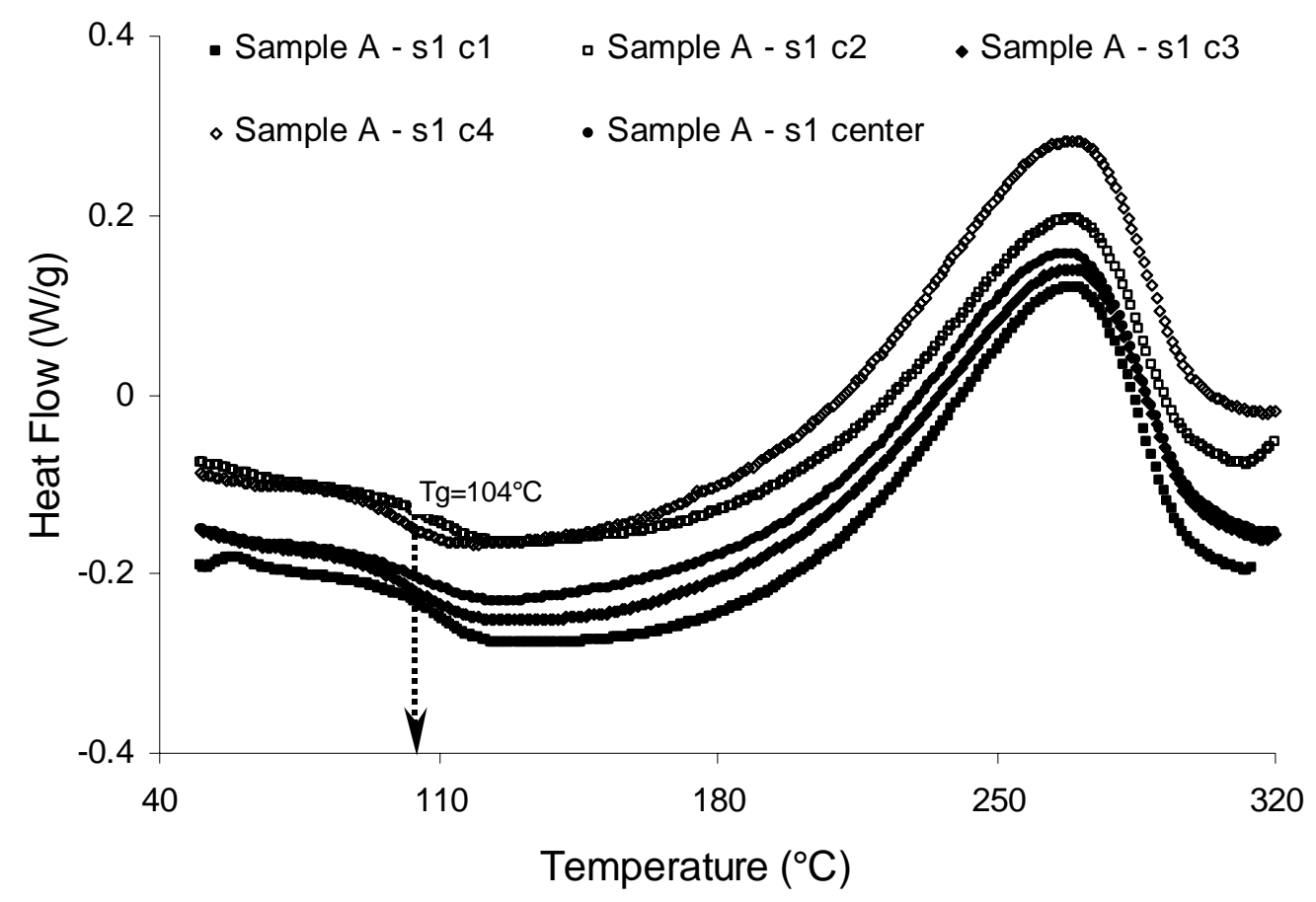

Fig. 3 Heat flow diagrams obtained by DSC measurements on specimens taken from the four corners and the centre position of resin plate A $(\alpha=0.68)$. Sample.. - s..- c.., indicates the type of sample (Sample), the side (s) from and the corner (c) from which the DSC samples were taken from.

\begin{tabular}{|c|c|c|c|}
\hline Plate Label & $\mathbf{T}_{\mathbf{g}}\left({ }^{\circ} \mathrm{C}\right)$ & $\begin{array}{c}\text { Residual Heat of } \\
\text { Reaction }(\mathrm{J} / \mathrm{g})\end{array}$ & Degree of Conversion \\
\hline A & $104 \pm 5$ & $132 \pm 3$ & 0.68 \\
\hline B & $133 \pm 3$ & $83 \pm 3$ & 0.80 \\
\hline C & $151 \pm 3$ & $54 \pm 3$ & 0.87 \\
\hline D & $165 \pm 3$ & $43 \pm 2$ & 0.90 \\
\hline E & $182 \pm 3$ & $18 \pm 1$ & 0.96 \\
\hline
\end{tabular}

Table 1 Average results of thermal analysis tests performed on samples taken from the partially cured plat 


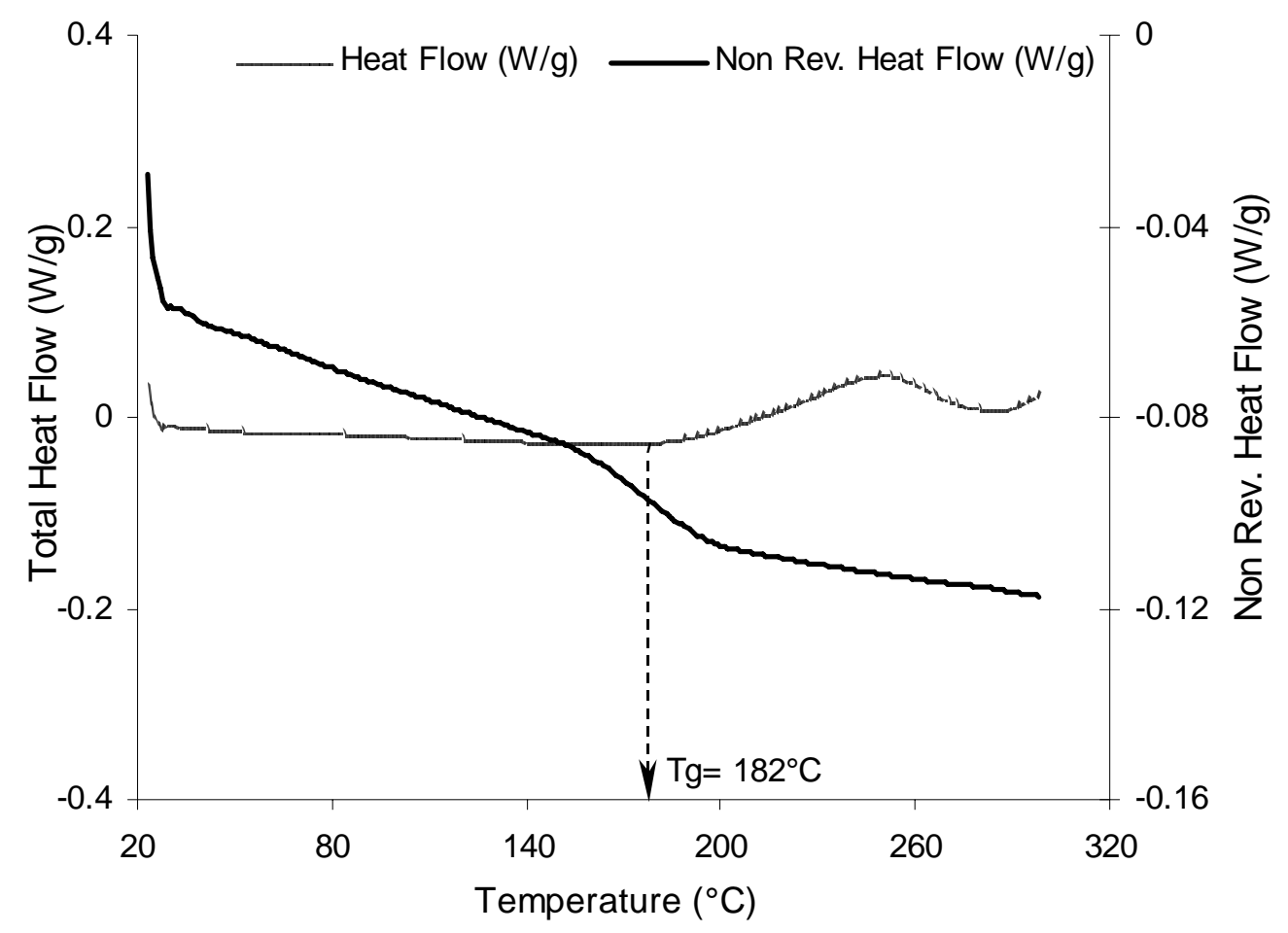

Fig. 4 Reversible and non-reversible heat flow curves for sample taken from resin plate E

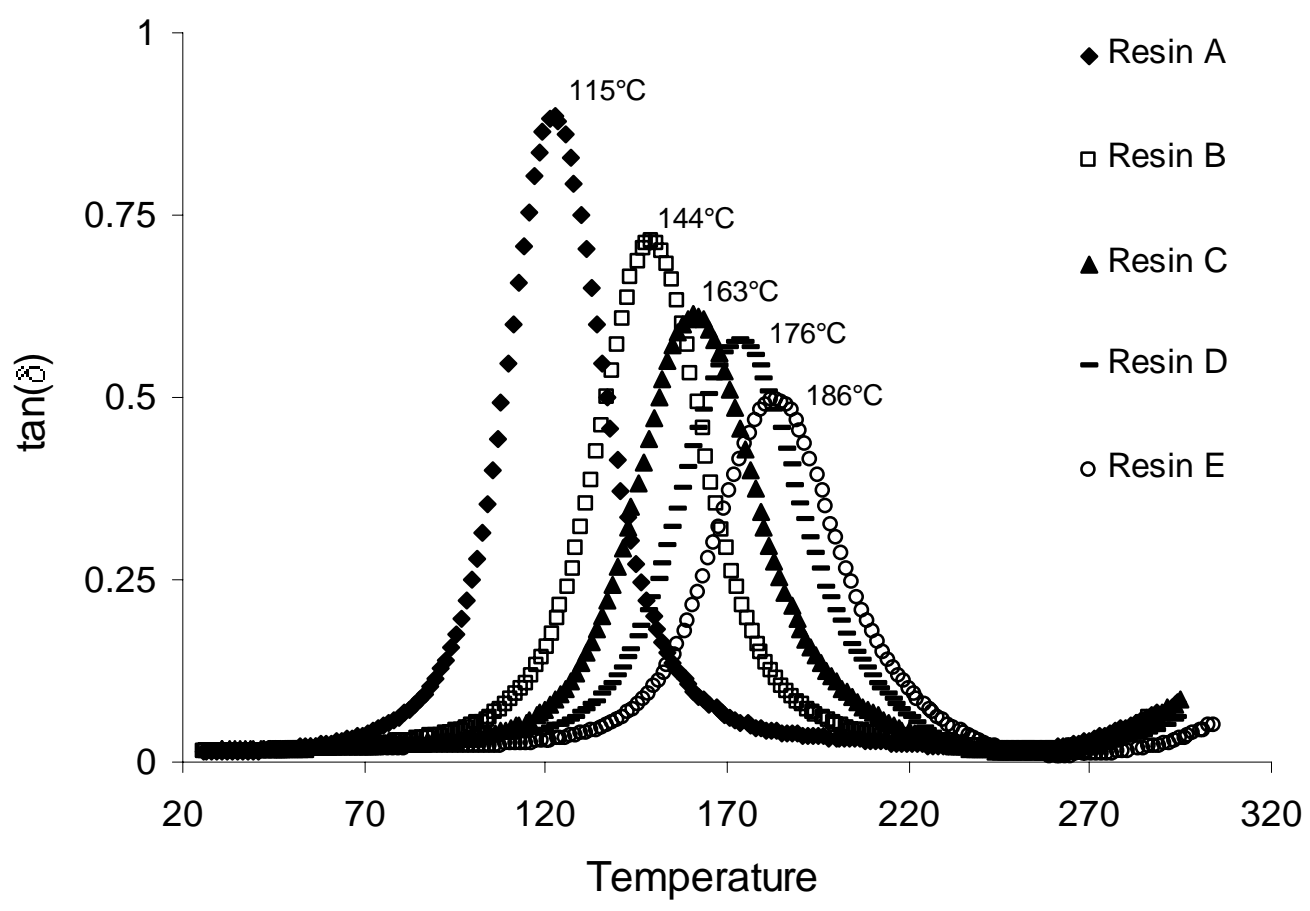

Fig. 5 Torsional rheometric results for partially cured samples. Glass transition temperature values are also reported. 


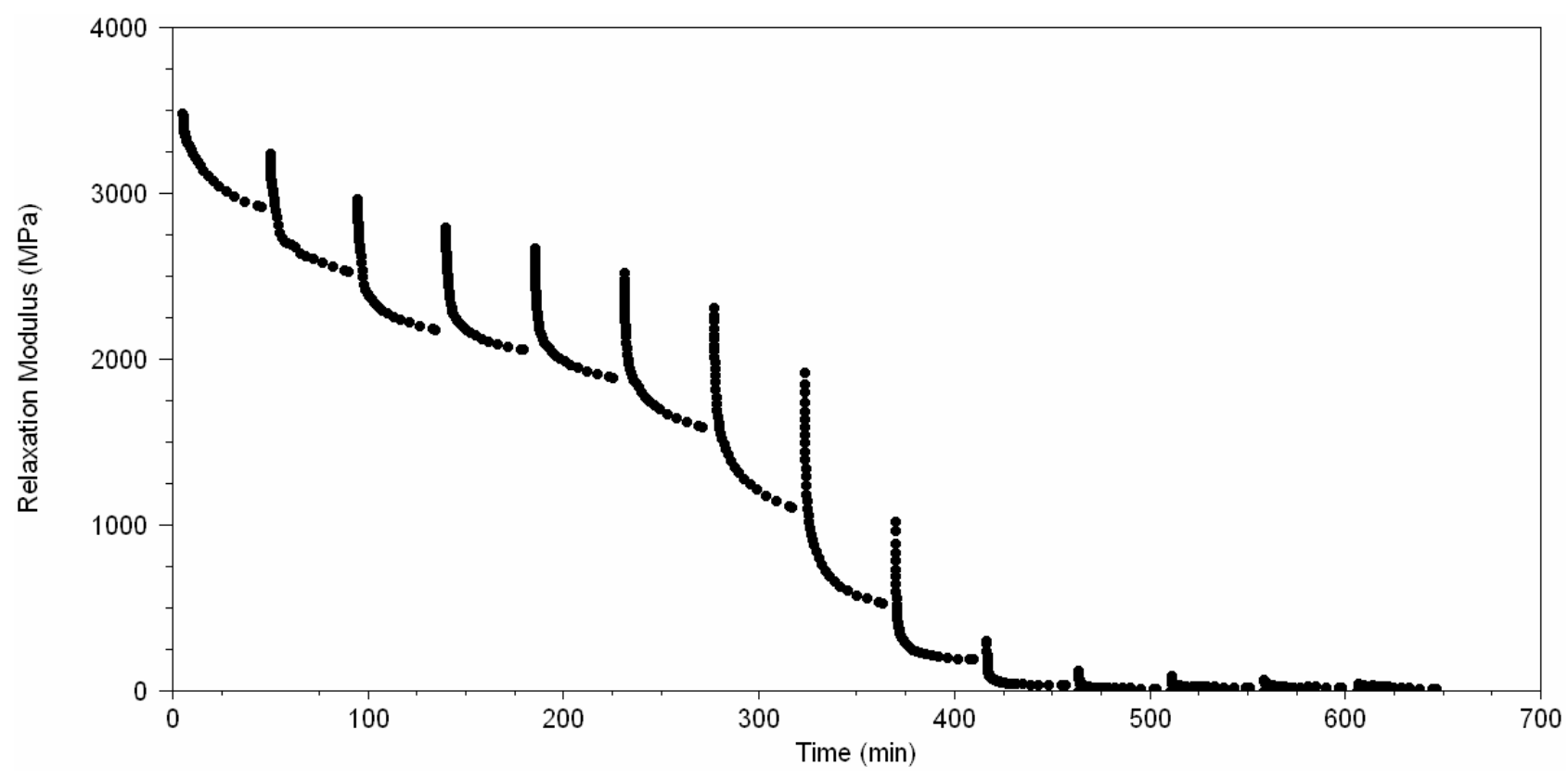

Fig. 6 Raw data of relaxation test on specimen type $\mathrm{D}(\alpha=0.90)$

- Relaxation Modulus (GPa) - Resin A - Temperature $\left({ }^{\circ} \mathrm{C}\right)$ - Resin A

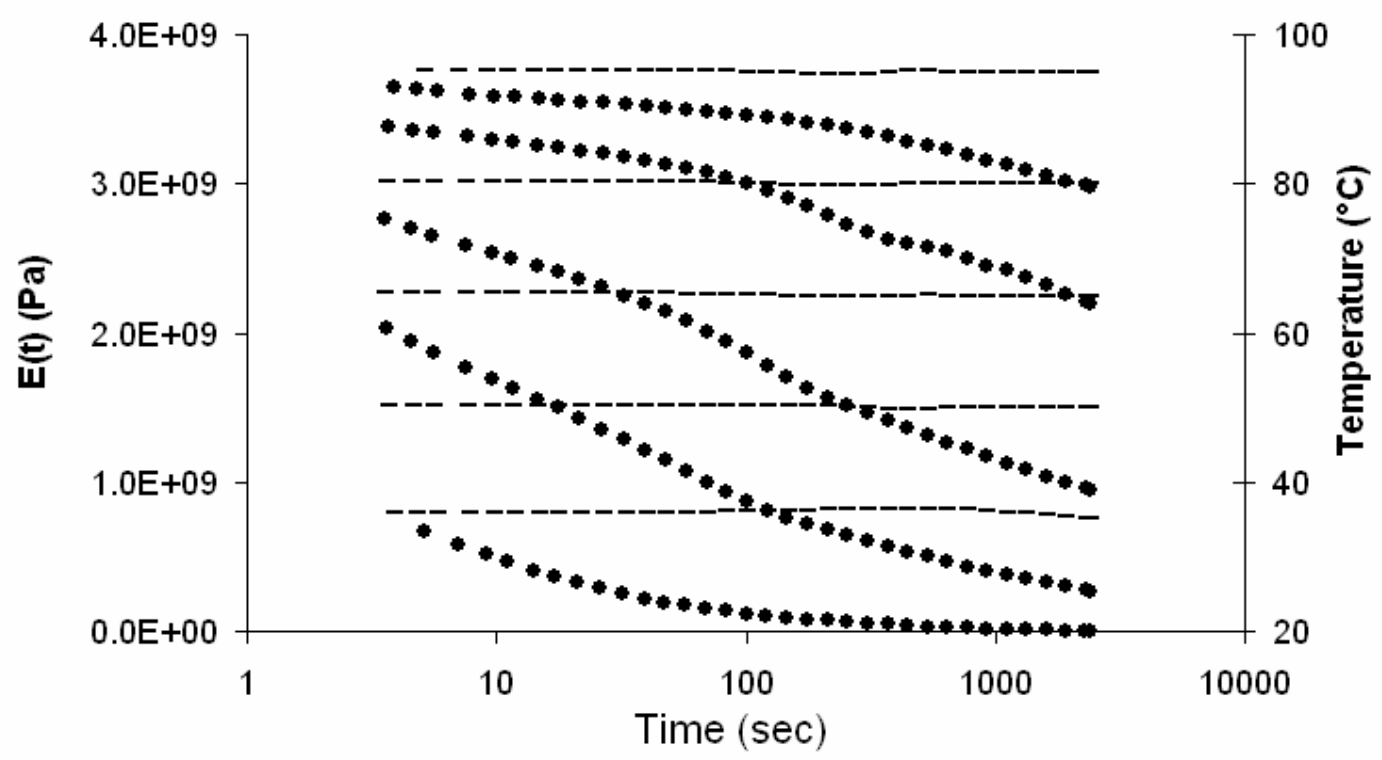

Fig. 7 Stress relaxation profiles at various temperatures for resin plate $A(\alpha=0.68)$ 

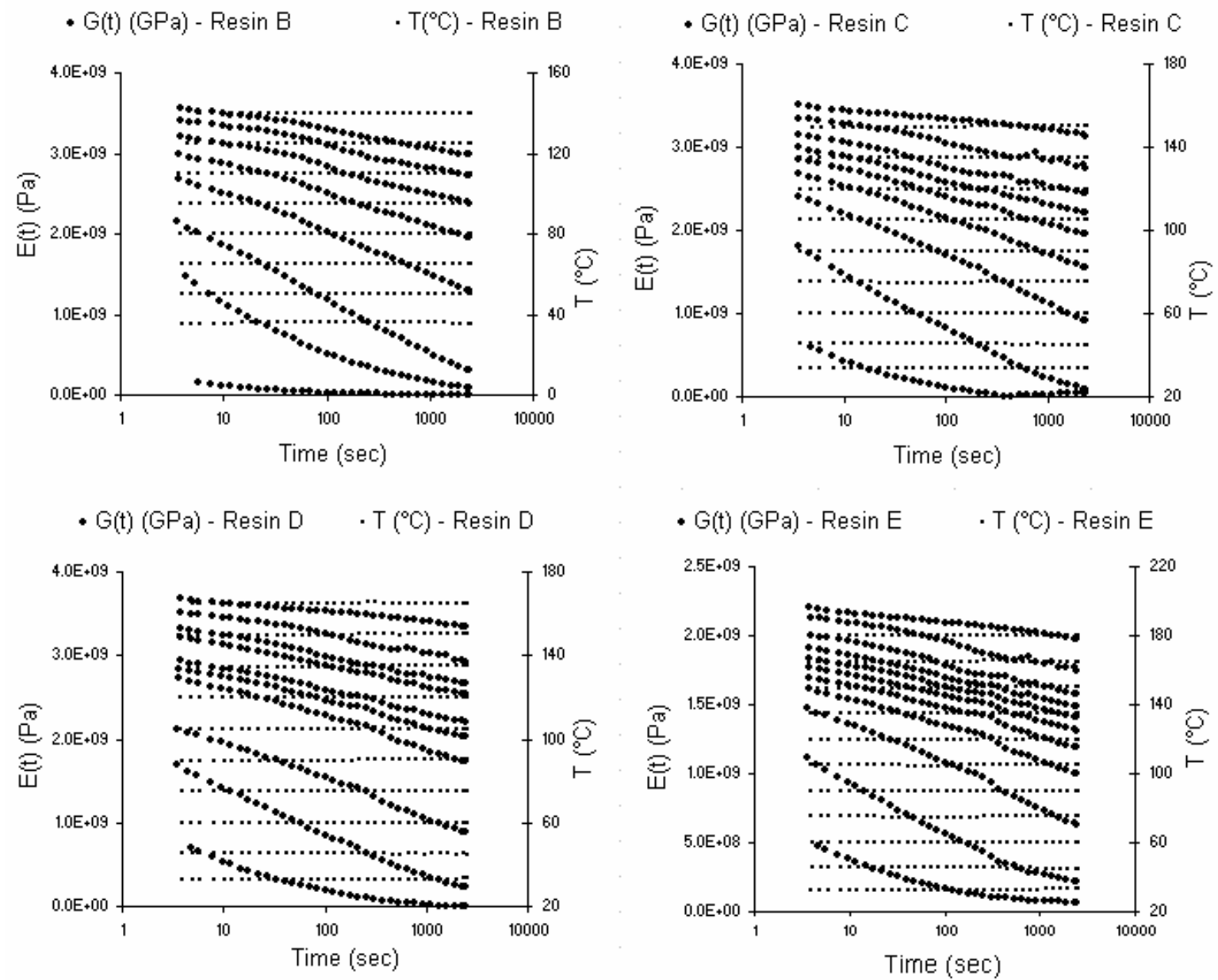

Fig. 8 Stress relaxation profiles at various T for resin plates B-C-D-E $(\alpha=0.80-0.87-0.90-0.96)$

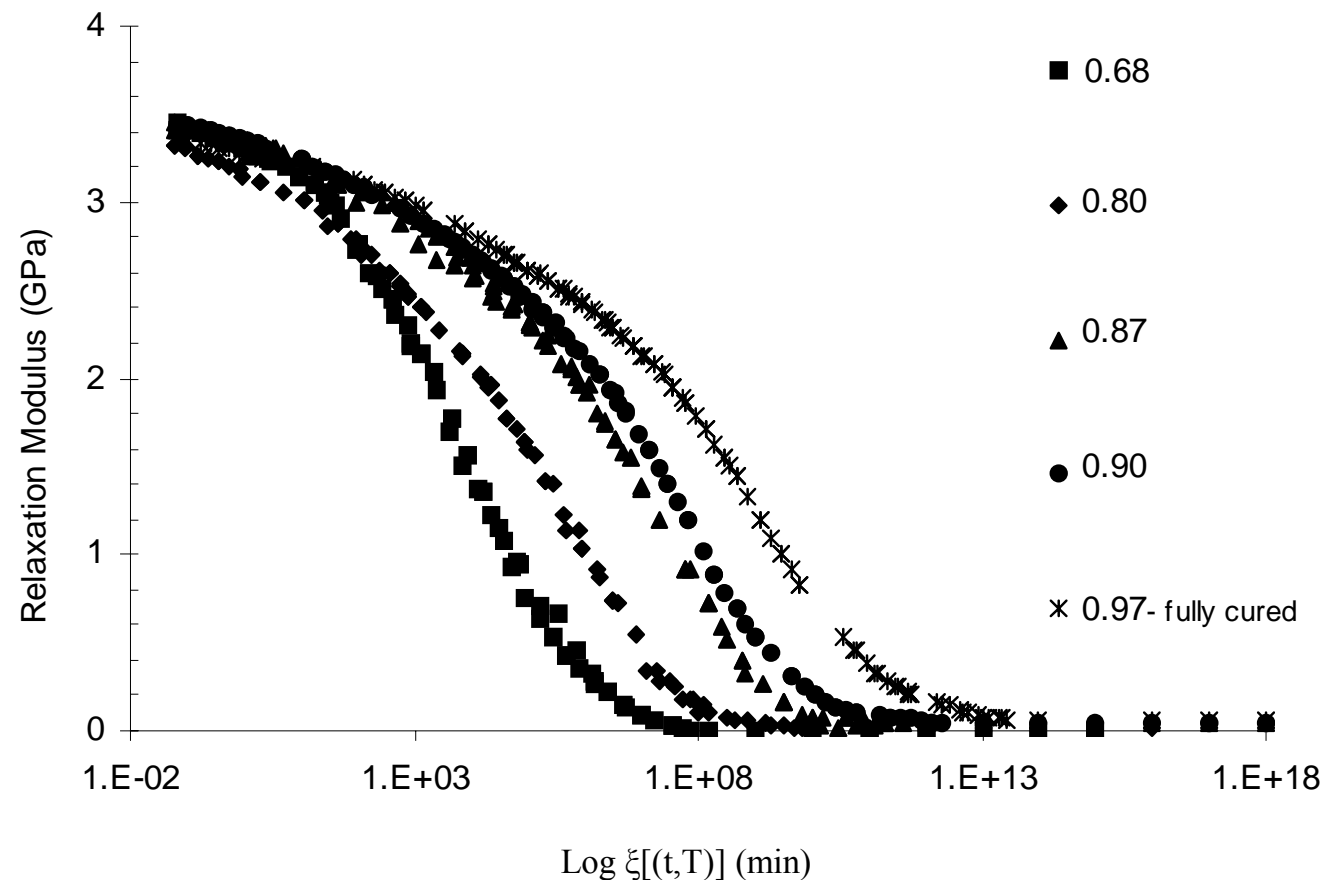

Fig. 9 - Experimentally obtained Master curves for all partially cured sample 


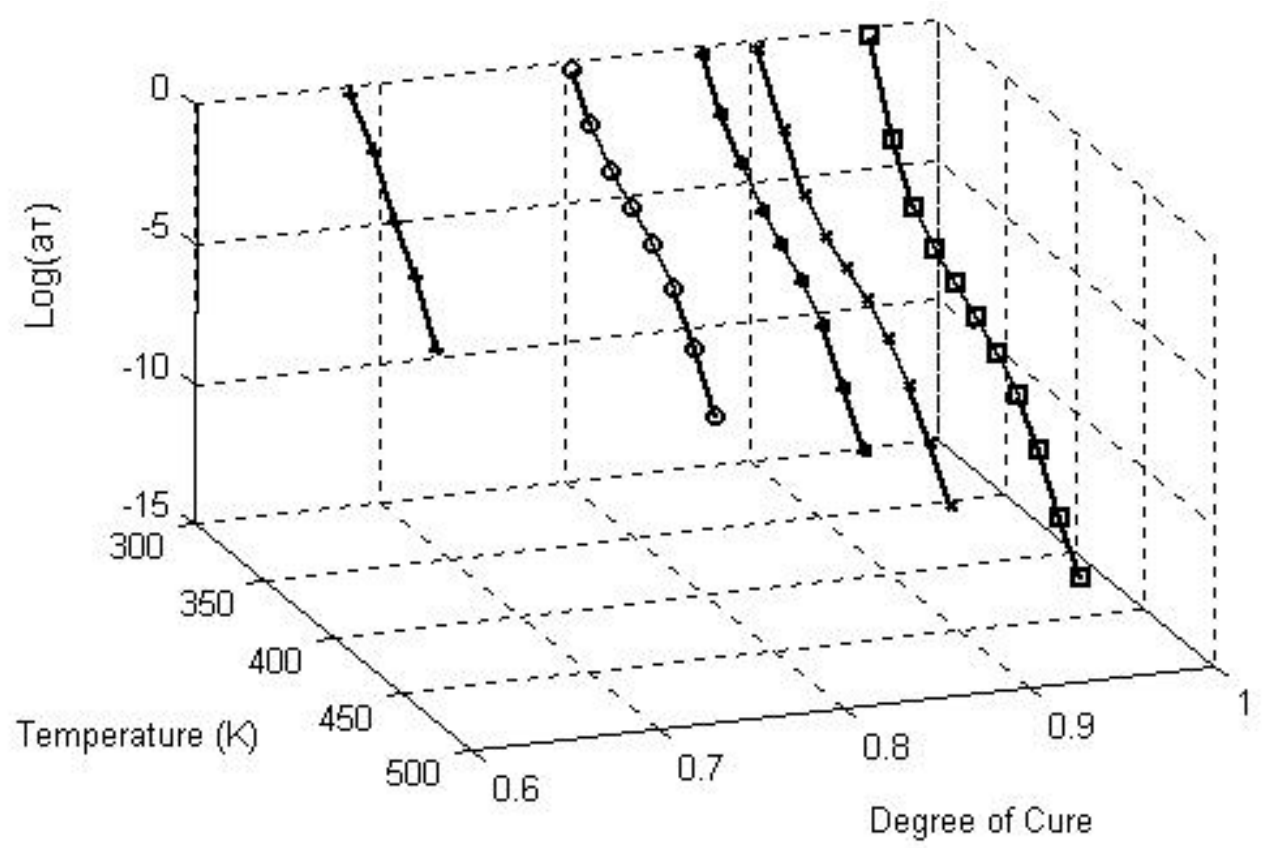

Fig. $103 \mathrm{D}$ plot of experimental shift factors vs. temperature and degree of cure

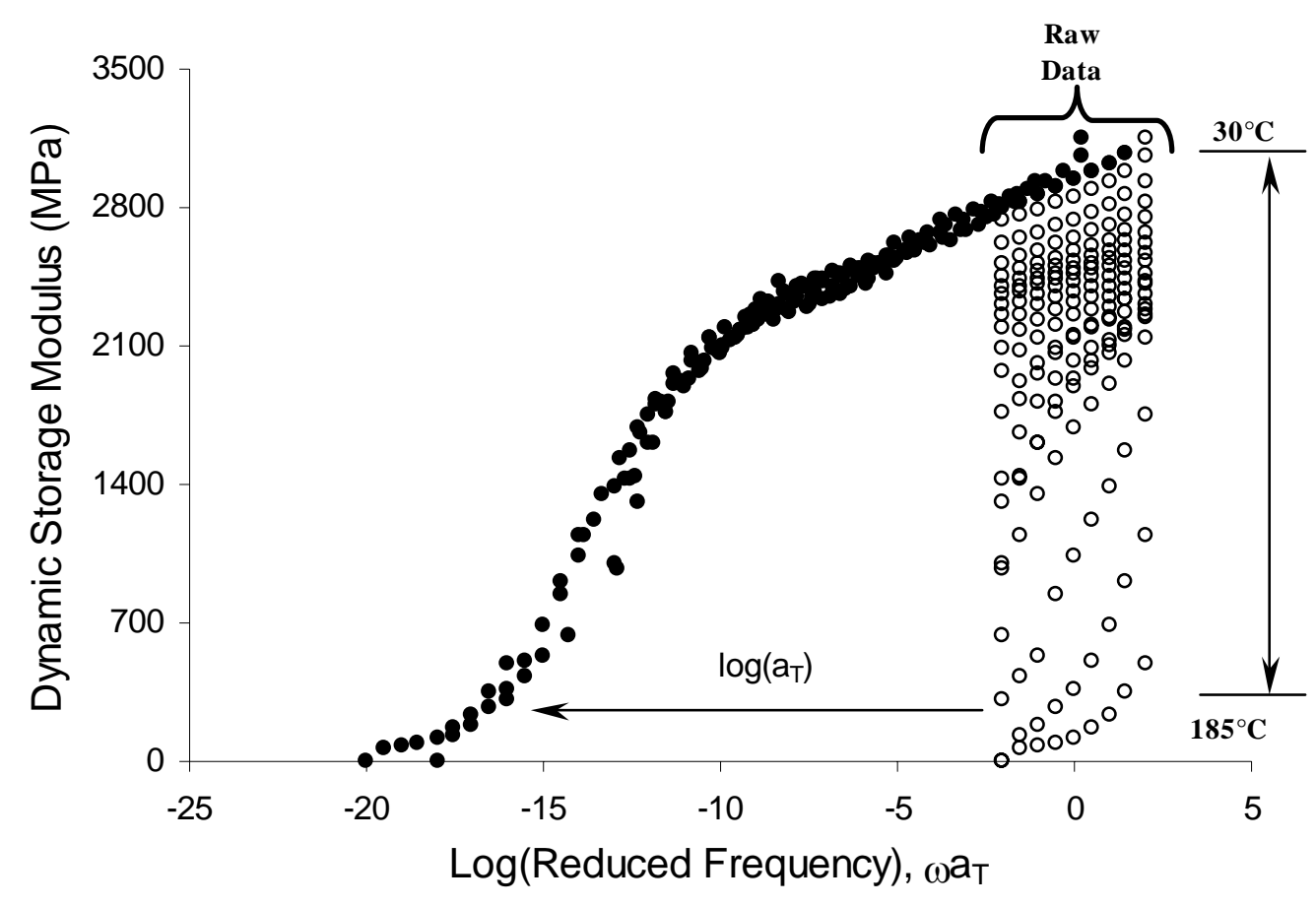

Fig. 11 Dynamic storage modulus master curve (sample E) obtained applying the shift factors from static tests 


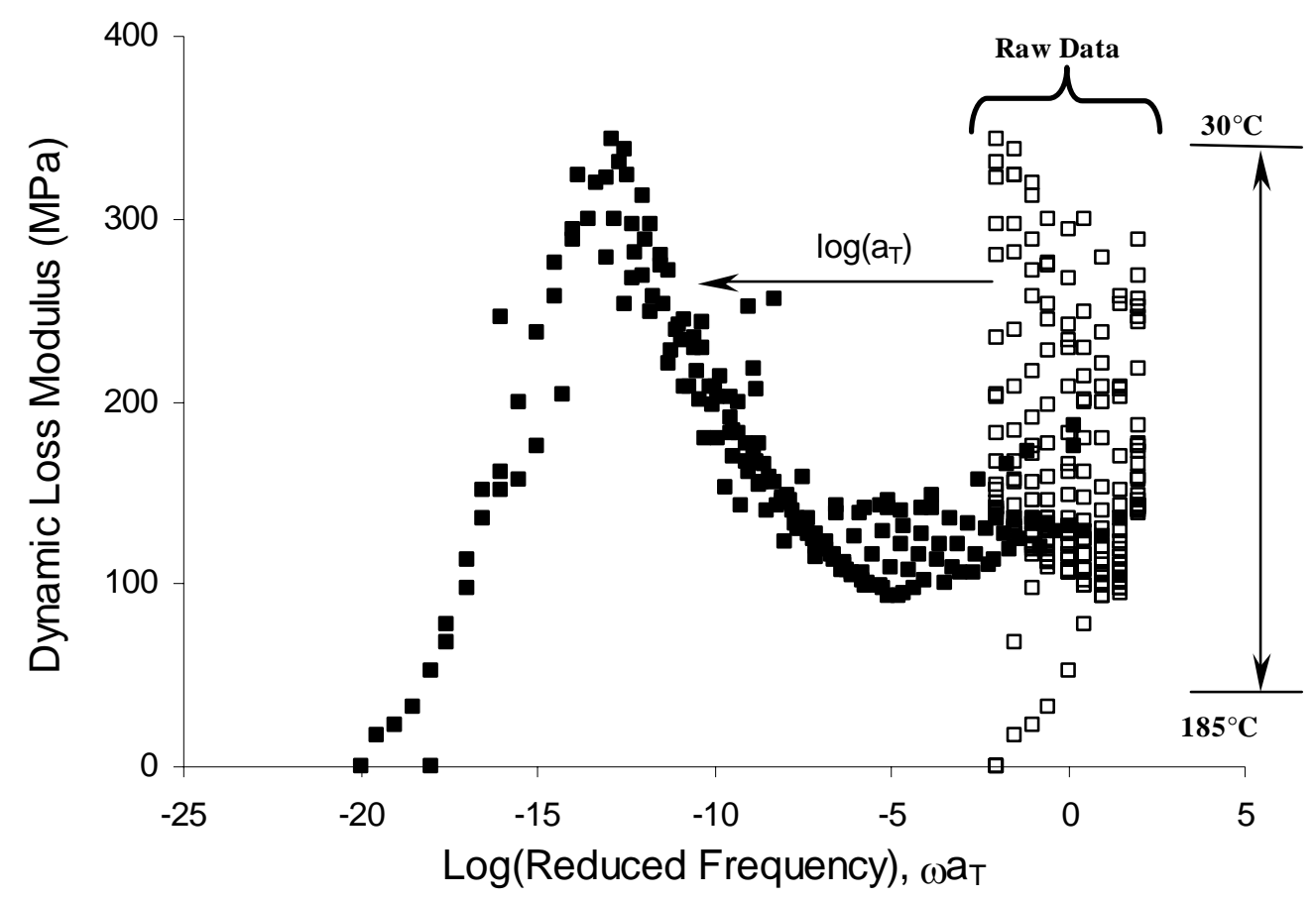

Fig. 12 Dynamic loss modulus master curve for sample plate E, applying shift factor values as obtained from relaxation modulus master curves

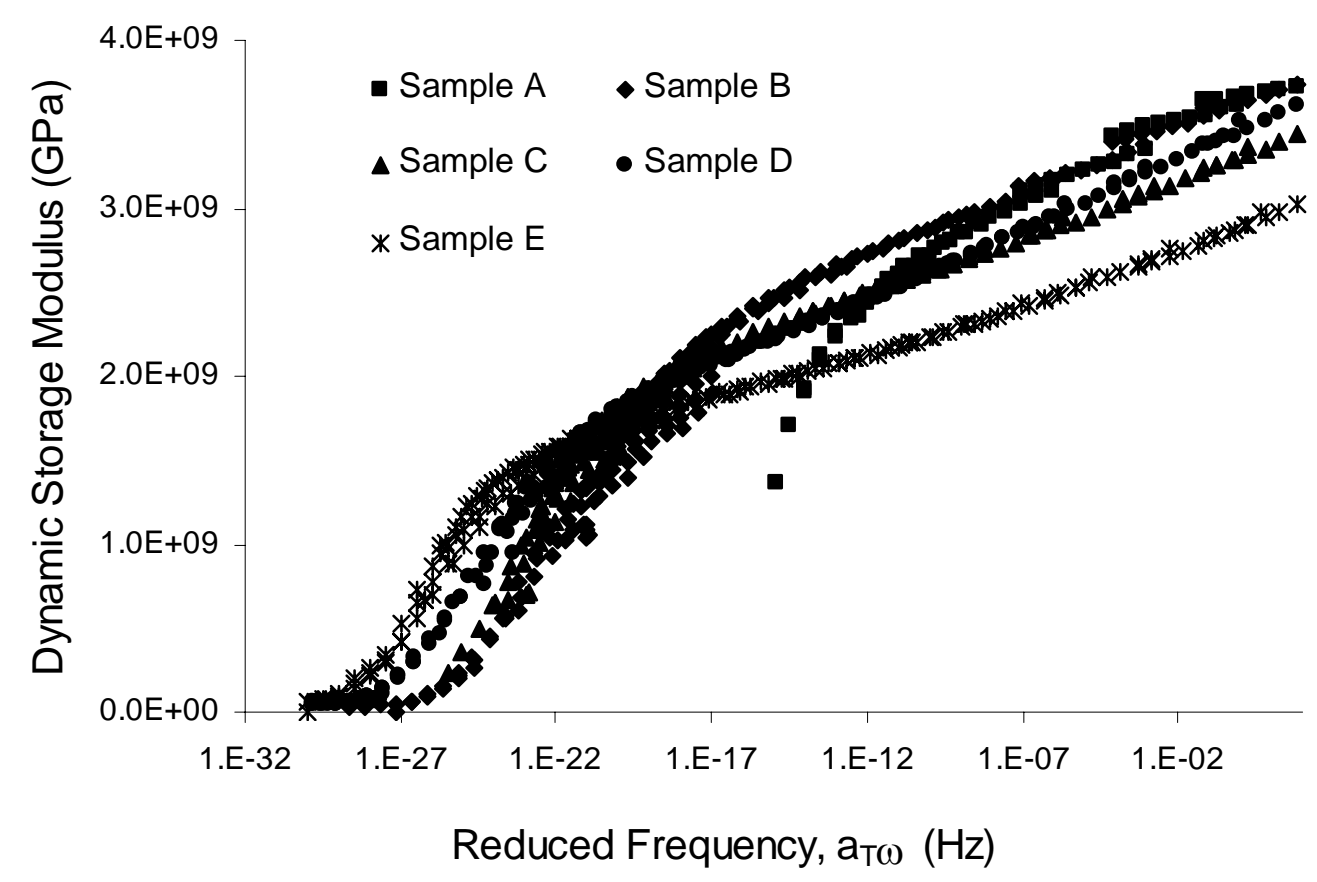

Fig. 13 Master curves of dynamic loss modulus for all the levels of conversion, constructed using values of the shift factor values obtained from master curves for the static stress relaxation tests. 


\section{Reference List}

1 S. A. Bidstrup and C. W. Macosko "Chemorheology Relations for Epoxy-Amine Crosslinking” Journal of Polymer Science: Part B: Polymer Physics, 28, 691-709, (1990)

2 S. R. White and P. T. Mather "A Comparison of Ultrasonic and Thermal Cure Monitoring of an Epoxy" Resin. Composite Polymers, 4, No. 6, 403-422, (1992)

3 J. O. Simpson and S. A. Bidstrup, "Rheological and Dielectric Changes during EpoxyAmine Cure", Journal of Polymer Science: Part B: Polymer Physics, 33, 55-62, (1995)

4 K. Suzuki, Y. Miyano and T. Kunio, "Change of Viscoelastic Properties of Epoxy Resin in the Curing Process", Journal of Applied Polymer Science, 21, 3367-3379, (1977)

5 K.S. Kim and H.T. Hahn, "Residual stress development during processing of graphite/epoxy composites", Composites Science and Technologies, 36, 121, (1989)

6 Y.K. Kim and S.R White, "Stress Relaxation Behavior of 3501-6 Epoxy Resin During Cure", Polymer Engineering and Science, 36, No. 23, 2852, (1996)

7 Y.K. Kim and S.R White, "Process-Induced Stress Relaxation Analysis of AS4/3501-6 Laminate", Journal of Reinforced Plastics and Composites, 26,No. 2, (1997)

8 S.L. Simon, G.B. MecKenna and O. Sindt, "A Thermo-Viscoelastic Model for the Mechanical Properties of an Epoxy during Cure", Journal of Applied Polymer Science, 76, No. 4, 495, (2000)

9 Y. Eom, L. Boogh, V. Michaud, P. Sunderland and J.A. Manson, "Cure kinetics and shrinkage model for epoxy-amine systems", Polymer Engineering and Science, 40, No.6, 1281, (2000)

10 D.J. O'Brien, P.T. Mather and S. R. White, "Viscoelastic Properties of an Epoxy Resin during Cure", Journal of Composite Materials, 35, 883, (2001)

11 Zarrelli, M., Skordos, A.A., Partridge, I.K, "Investigation of cure induced shrinkage in unreinforced epoxy resin”, Plastics, Rubber and Composites 31, (9), (2002)

12 Zarrelli M., in "Cure induced property changes and warpage in thermoset resin and composites, PhD Thesis, Cranfield University, UK (2003 